\title{
Theoretical and Empirical Evidence for Extended Phenotypes in a Specialized Parasite of Spiders
}

\author{
Stephen James Saltamachia ${ }^{1}$ \\ ${ }^{1}$ University of Louisiana at Lafayette
}

February 28, 2022

\begin{abstract}
Parasite induced changes in host behavior is considered adaptive if it provides a reproductive advantage to the parasite. The entomopathogenic fungi are a fascinating group of arthropod parasites that exhibit enormous diversity in terms of both host choice and reproductive strategy, some of which actively manipulate the behavior of their host as a means to increase reproductive success. Here we focus on anamorphs of the genus Torrubiella, namely Gibellula spp., which are all obligate pathogens of spiders that kill their hosts in locations suspiciously ideal for fungal reproduction and propagation. In a final, eerily purposeful act, hosts reliably spin a thin stratum of web on the under-surface of a leaf to which they secure themselves immediately before death. We provide both theoretical and empirical lines of evidence that this phenomenon represents an extended phenotype of parasite genes analogous to those observed in other taxonomically related parasite-host systems. We argue that observations of spiders killed by Gibellula sp. both in the field (in situ) and the laboratory (in vitro), as well as this species' close phylogenetic relation to other fungal lineages believed or suspected to influence host behavior, are strong evidence for the presence of adaptive manipulation.
\end{abstract}

\section{Introduction}

Parasitic life strategies dominate biological systems and can profoundly influence several aspects of host biology, ecology, and community structure (Windsor 1989; Jones et al. 1994; Thomas et al. 1999; Moore 2002; Thomas et al. 2005; Peay et al. 2008; Anderson et al. 2009; Pontoppidan et al. 2009). Several studies have demonstrated a propensity for certain fungal lineages to actively manipulate their hosts through complex changes in behavior and body plan, and these results have captured the attention and imagination of evolutionary ecologists in recent years (Moore 2002; Thomas et al. 2005; Anderson et al. 2009; Pontoppidan et al. 2009; Goodman and Johnson 2011; Hughes et al. 2011; Hughes et al. 2016). The majority of these studies have focused primarily on myrmecophilous Ophiocordyceps species, namelyO. unilateralis , for their particularly spectacular influence on their ant-hosts (Anderson et al. 2009; Hughes et al. 2011). Parasite induced changes in host physiology force ants infected with $O$. unilateralis to permanently secure themselves in locations specifically suited to fungal development and dispersal via irreversible "death-grip" behavior (Andersen et al. 2009; Hughes et al. 2011; Weinersmith and Faulkes 2014; Thomas et. al. 2005, 2010). Scores of ants killed in this way tend to aggregate into so-called "graveyards", a phenomenon thought to represent an "extended phenotype" of parasite gene expression (Dawkins 1982; Anderson et al. 2009; Pontoppidan et al. 2009). In the context of parasitic manipulation of host behavior, an extended phenotype refers to the influence of parasite gene expression on host phenotype, particularly when this behavioral manipulation serves to increases parasite transmission and fitness (Dawkins 1982; Hughes et al. 2011; Bailey 2012). Such dramatic examples of extended phenotypes are not limited to Ophiocordyceps species, however, and empirical evidence suggests that the same phenomena may occur among certain specialized fungal parasites of spiders.

\section{The parasite-host system}


Asexual morphs of the hyphomycete genera Torrubiella arachnophila(Hypocreales, Ascomycota), namely Gibellula spp ., are specialized fungal pathogens attacking primarily members of the Salticidae family of spiders (Mains, 1950; Samson and Evans, 1982, 1992). These fungi are found globally throughout the tropics and sub tropics and range into temperate climates (Evans and Samson 1987). Two species occur in North America, G. pulchra and G. leiopus (Mains 1950), the former known from as far as north as Nova Scotia, Canada (Strongman, 1991). These fungi play an important role in the regulation and maintenance of arthropod populations, especially in tropical ecosystems where they may constitute a significant mortality factor of spiders (Evans 1982; Evans and Samson 1987; Augustyniuk-Kram and Kram 2012).

Immediately prior to death, spiders infected with the species Gibellula pulchra spin a thin flat layer of webbing on the underside of a leaf well above the forest floor, then situate themselves upon the web as if resting. The host dies shortly after taking this final position and the emergence of fungal mycelia from the feet of the host further secure the cadaver to the webbing. The fungal hyphae do not penetrate plant tissues and the leaves on which hosts die are typically healthy and without damage or leafspot (Selçuk et al. 2004). In the following days and weeks, whirling stalks of fungal hyphae bearing thousands of conidia emerge from the mummified cadaver, allowing huge numbers of the infectious propagules to disperse with wind and minor perturbation (Figure 1).

Securing of hosts to the substrata is a common feature in the final stages of many fungal entomopathogen life cycles (Hughes et al. 2010; Malagocka et al. 2015; Hughes et al 2016; Araújo and Hughes 2016). However, an interesting distinction in the case of spiders infected with Gibellula pulchra is that the "death-bed" woven by the spider constitutes the substrata rather than the leaf surface. As with the "death-grip" behavior observed in Ophiocordyceps- infected ants (Anderson et al. 2009; Hughes et al. 2011), this peculiar "death-bed" behavior is not among the repertoire of web-structures built by healthy uninfected individuals, and ostensibly serves no purpose other than securing the host post-mortem. This characteristic pre-death ritual is also observed both in situ in field specimens and in laboratory specimens infected under controlled conditions. It is therefore argued that this behavior represents an extended phenotype of parasite gene expression, optimizing parasite fitness by ensuring that the hosts die securely in locations ideally suited for dispersal. Furthermore, since the act of spinning the deathbed in such specific locations represent novel behaviors absent in uninfected hosts, and which clearly benefit fungal dispersal, it is also argued that this manipulation of host behavior is adaptive for the parasite.

\section{Materials and Methods}

\section{Collection and Isolation}

A spider infected by the fungus Gibellula pulchra was collected in May 2015, from a bottomland hardwood forest near Lafayette, Louisiana $\left(30^{\circ} 15^{\prime} 28.7^{\prime \prime} \mathrm{N}, 91 \operatorname{deg} 59^{\prime} 17.6^{\prime \prime} \mathrm{W}\right)$. The cadaver was collected along with the leaf to which it was attached and transported to the lab in a clean plastic container (Figure 1e).

The fungus was isolated on Sabouraud Dextrose Agar plus 2\% yeast extract (SDAY+) by holding the specimen over the open plate and gently tapping the leaf to which the infected spider was attached, causing fungal spores to be released onto the surface of the medium. Cultures were maintained at room temperature and ambient light and checked daily for fungal growth. Subcultures were made from the germinating spores and pure cultures were established and used for isolation of genomic DNA. The ITS region was sequenced (primers; ITS1F and ITS4) to further confirm the identity of the fungus.

The host was difficult to identify due to the extent of fungal growth, though this fungus has been recovered from several common species in the area including Colonus spp ., Anasaitis canosa, andPlatycryptus sp .. Due to their commonality, individuals of the genus Colonus (previously Thiodina sp.) were chosen as hosts for an ad hoc experiment intending to confirm the cause of mortality as being due to the fungus G. pulchra

\section{Experimental Procedures}

5 presumably healthy individuals were collected from the same location as the infected cadaver, and consisted 
of 3 male and 2 female. The spiders were housed individually in a petri dishes, each containing a rolled up piece of filter paper (for shelter) and a small moistened cotton ball (for drinking and humidity).

To infect the spiders, flies were captured and placed in the refrigerator for approximately 1 minute (too long and they die, too short and they fly away) to briefly disable them. Using forceps to grab the fly by the wings, the insects were then brushed against the infected cadaver containing infectious conidia. After inoculating the fly with conidia it is immediately placed in the petri dish containing the healthy spider host. The spider graciously accepts the meal and is subsequently inoculated with conidia from the surface of the fly. This process was done with 3 of the 5 spiders collected as the other 2 would serve as a control. No preparations to measure the amount of inoculum were made since the purpose of the experiment was simply to demonstrate that G. pulchra is indeed a lethal pathogen causing the observed phenotype.

\section{Results and Discussion}

In a fascinating testament to the prevalence of parasites and the pressure imposed on their hosts, 2 of the 5 spiders collected for the experiment succumbed to infections apparently already present at the time of collection. One control female and one experimental male died within 2-3 days of collection, the former quickly sprouting a large horsehair worm-like parasite and the latter produced the fungusPurpureocillium atypicola ( formerly Nomurea atypicola), another spider-specific ascomycete of the family Ophiocordycipitaceae (Figure 2a). The two surviving experimental hosts died after several weeks, affixing themselves to the underside of the petri lid (Figure 2b-c). The hosts were then incubated in place by sealing the plate containing the cadaver and moistened cotton ball with paraffin film (Figure 2d). The subsequent fungal growth was then subcultured and compared to the original isolate (Figure 3). Comparisons based on both microscopic examination of conidiogenous structures as well as macroscopic colony growth characteristics demonstrate Gibellula pulchra as the cause of death. The remaining control spider was eventually set free at the conclusion of the experiment.

\section{Important considerations for adaptive vs. non-adaptive manipulation}

Parasitic manipulation of host behavior is not always adaptive. Both direct and indirect mechanisms can lead to increased parasite transmission (Adamo 2002) and making the distinction between 'advantageous by-products' of infection and true adaptive manipulation requires an understanding of which mechanisms are involved (Poulin 1995, 2010; Thomas et al. 2005). In effort to deal with this, Poulin (1995) synthesized four criteria by which one can theoretically distinguish between 'true' adaptive traits and advantageous 'side-effects'. The first criteria regard "complexity", considering that complex traits are unlikely to arise by chance or accident and require an organizing principle like natural selection. Poulin (1995) acknowledges the difficulty in assessing these criteria since even simple changes in behavior can result from complex mechanisms. Nevertheless, the behavior presented in this paper whereby the host spider is manipulated to perform a task serving the post-mortem needs of the parasite, is indicative of a complex mechanism refined by selection. The second criteria is of "purposiveness of design", referring to characters or traits seemingly "too well fitted" to fulfil a certain function to have arisen by chance. Such observations of apparent conformity between purpose, design, and function, used to be presented as evidence of divine creation, but are now seen as strong evidence of adaptation through selection (Bekoff and Allen 1995). The locations of host spiders killed by Gibellula spp. certainly appear to serve an obvious purpose by ensuring that the host is in an elevated and protected position for the dispersal of conidia. The third criterion is regarding the "convergence", or independent origins of an adaptive trait in the phylogenetic lineage of a group of organisms and is seen as a "strong" indicator that the trait is not an accidental side-effect (Allen and Bekoff 1995). Poulin (1995) stipulates that traits do not have to be identical but only analogous, performing the same function (i.e. dispersal). As mentioned above, the closely related genusOphiocordyceps contains a huge assemblage of ant-pathogenic species wherein hosts secure themselves to the substrata via irreversible deathgrip behavior immediately prior to death. This behavior, we argue, is analogous to the deathbed behavior of infected spiders. The final and most important of the four criteria according to Poulin (1995) are the "fitness effects", though he admits that measuring fitness is logistically difficult and not on its own conclusive since even undetectable fitness advantages can be adaptive. As mentioned above, the specificity of these death 
locations ostensibly serves no other purpose than to maximize fungal spore dispersal and thus, parasite fitness.

\section{Conclusions and other considerations towards a potential model}

-The deathbed behavior of spiders infected with Gibellula spp.likely represents an extended phenotype of parasite gene expression. Parasite induced changes in this host-pathogen system fulfil all four criteria set forth by Poulin (1995) to assess if the observed manipulation is 'truly' adaptive for the parasite. However, web building prior to death is not ubiquitous among spiders infected by Torrubielloid anamorphs and other species in this genus exhibit similar phenotypes but with characterizable differences in host choice and death location. For example, another Torrubielloid anamorph (Gibellula leiopus ) from the same area infecting the spider Trachelas tranquillus, invariably cause hosts to die on elevated tree branches and never on leaves. In this species, a fungal mat appears to form the only attachment of the host spider to the tree surface. Nonetheless, this behavior of dying in elevated (1-3 meters) locations on the underside of tree branches also provides fitness benefits for the parasite via efficient dispersal of infectious propagules (Araujo and Hughes 2016). The differences in host death locations between these two related pathogens likely reflects the differences in the specific ecologies of the host species, and like O. unilateralis infected ants, may be indicative of adaptive manipulation by the parasite.

Parasitized hosts dying on the underside of leaves is a common feature in the life cycle of many entomopathogenic fungi and represents a clear fitness advantage for the parasite (Araujo and Hughes 2016). The locations where infected hosts die can be phylogenetically informative for some highly specialized species and relates directly to the specific ecological niche exploited by the host (Evans et al. 2018, Araujo et al. 2018). The Torrubielloid anamorphs such as previously mentioned species of Gibellula appear to follow the same trend, though no studies have yet explored specialized fungal pathogens of spiders with similarly comprehensive detail. Despite compelling empirical evidence, the current literature is bereft of any experimental support for adaptive manipulation in this particular parasite-host system. These specialized fungal parasites of spiders may therefore provide a valuable experimental model for further exploring the dynamics of parasitic manipulation since rearing of large numbers of hosts in the laboratory is fairly simple and low cost. Such models will endeavor to better define the elusive distinction between adaptive and non-adaptive manipulation, as it will likely provide valuable insight into the evolution of specialized parasite-host systems.

\section{References}

Adamo, S.A., 2002. Modulating the modulators: parasites, neuromodulators and host behavioral change. Brain, behavior and evolution, 60 (6), pp.370-377.

Allen, C. and Bekoff, M., 1995. Biological function, adaptation, and natural design. Philosophy of Science ,62 (4), pp.609-622.

Andersen SB, Gerritsma S, Yusah KM, Mayntz D, Hywel-Jones NL, et al. (2009) The life of a dead ant: the expression of an adaptive extended phenotype. Am Nat 174: 424-433. pmid:19627240

Andersen, Sandra B., et al. "Disease dynamics in a specialized parasite of ant societies." PLoS One 7.5 (2012): e36352.

Anna Augustyniuk-Kram and Karol J. Kram (2012). Entomopathogenic Fungi as an Important Natural

Regulator of Insect Outbreaks in Forests (Review), Forest Ecosystems - More than Just Trees, Dr Juan A. Blanco (Ed.), ISBN: 978-953-51-0202-1

Araujo, J.P. and Hughes, D.P., 2016. Diversity of entomopathogenic fungi: which groups conquered the insect body?. In Advances in genetics (Vol. 94, pp. 1-39). Academic Press.

Araujo, J.P.M., Evans, H.C., Kepler, R. and Hughes, D.P., 2018. Zombie-ant fungi across continents: 15 new species and new combinations within Ophiocordyceps. I. Myrmecophilous hirsutelloid species. Studies in Mycology, 90 , pp.119-160. 
Bailey, N.W., 2012. Evolutionary models of extended phenotypes. Trends in ecology E6 evolution, 27 (10), pp.561-569.

Byers, John, Eileen Hebets, and Jeffrey Podos. "Female mate choice based upon male motor performance." Animal Behaviour 79.4 (2010): 771-778.

Dawkins, R. 1982. The extended phenotype. Oxford University Press, Oxford.

de Bekker, Charissa, et al. "Species-specific ant brain manipulation by a specialized fungal parasite." $B M C$ evolutionary biology 14.1 (2014): 166.

Evans, H. C., and R. A. Samson. "Cordyceps species and their anamorphs pathogenic on ants (Formicidae) in tropical forest ecosystems II. The Camponotus (Formicinae) complex." Transactions of the British Mycological Society 82.1 (1984): 127-150.

Evans, H. C. "Entomogenous fungi in tropical forest ecosystems: an appraisal." Ecological Entomology 7.1 (1982): 47-60

Evans, H.C., Elliot, S.L. and Hughes, D.P., 2011. Hidden diversity behind the zombie-ant fungus Ophiocordyceps unilateralis: four new species described from carpenter ants in Minas Gerais, Brazil. PloS one, 6 (3).

Evans, H.C., Araujo, J.P.M., Halfeld, V.R. and Hughes, D.P., 2018. Epitypification and re-description of the zombie-ant fungus, Ophiocordyceps unilateralis (Ophiocordycipitaceae). Fungal Systematics and Evolution , 1 (1), pp.13-22.

Goodman, Brett A., and Pieter TJ Johnson. "Disease and the extended phenotype: parasites control host performance and survival through induced changes in body plan." PLoS One 6.5 (2011): e20193.

Gupta, Gaurav, Steve Oghumu, and Abhay R. Satoskar. "Mechanisms of Immune Evasion in Leishmaniasis." Advances in applied microbiology 82 (2013): 155-184. PMC. Web. 26 Apr. 2017.

Hill, David E. "Predatory pursuit of ants by Anasaitis canosa (Araneae, Salticidae)." (2006).

Hughes, David P., et al. "Behavioral mechanisms and morphological symptoms of zombie ants dying from fungal infection." BMC ecology11.1 (2011): 13.

Hughes, D.P., Araujo, J.P.M., Loreto, R.G., Quevillon, L., De Bekker, C. and Evans, H.C., 2016. From so simple a beginning: the evolution of behavioral manipulation by fungi. In Advances in Genetics (Vol. 94, pp. 437-469). Academic Press.

Isaka, Masahiko, et al. "Bioactive substances from insect pathogenic fungi." Accounts of Chemical Research 38.10 (2005): 813-823.

Jones, C. G., Lawton, J. H. and Shachak, M. 1994. Organisms as ecosystem engineers. - Oikos 69: 373-386.

Johnson, Desiree, et al. "Systematics and evolution of the genus Torrubiella (Hypocreales, Ascomycota)." mycological research113.3 (2009): 279-289.

Kobmoo, Noppol, et al. "New species of Ophiocordyceps unilateralis, an ubiquitous pathogen of ants from Thailand." Fungal biology 119.1 (2015): 44-52.

Mains, Edwin B. "The genus Gibellula on spiders in North America." Mycologia 42.2 (1950): 306-321.

Małagocka, J., Grell, M.N., Lange, L., Eilenberg, J. and Jensen, A.B., 2015. Transcriptome of an entomophthoralean fungus (Pandora formicae) shows molecular machinery adjusted for successful host exploitation and transmission. Journal of invertebrate pathology, 128, pp.47-56.

Mongkolsamrit, S., et al. "Life cycle, host range and temporal variation of Ophiocordyceps unilateralis/Hirsutella formicarum on Formicine ants." Journal of invertebrate pathology 111.3 (2012): 217-224. 
Moore, J., 2002. Parasites and the behavior of animals . Oxford University Press on Demand.

Peay, K.G., Kennedy, P.G. and Bruns, T.D., 2008. Fungal community ecology: a hybrid beast with a molecular master. Bioscience, 58 (9), pp.799-810.

Pontoppidan, Maj-Britt, et al. "Graveyards on the move: the spatio-temporal distribution of dead Ophiocordyceps-infected ants." PloS one 4.3 (2009): e4835.

Poulin, R. "Manipulation of host behaviour by parasites: a weakening paradigm?." Proceedings of the Royal Society of London B: Biological Sciences 267.1445 (2000): 787-792.

Poulin, R., Fredensborg, B.L., Hansen, E. and Leung, T.L., 2005. The true cost of host manipulation by parasites. Behavioural Processes, 68 (3), pp.241-244.

Richman, David B. "Epigamic display in jumping spiders (Araneae, Salticidae) and its use in systematics." Journal of arachnology(1982): 47-67.

Schmid-Hempel, Paul. "Parasite immune evasion: a momentous molecular war." Trends in Ecology $\mathcal{G}^{\circ}$ Evolution 23.6 (2008): 318-326.

Selçuk, Faruk, Elşad Hüseyin, and Muhammet Gaffaroğlu. "Occurence of the araneogenous fungus Gibellula pulchra in Turkey." Mycologia Balcanica 1.1 (2004): 61-62.

Strongman, D.B., 1991. Gibellula pulchra from a spider (Salticidae) in Nova Scotia, Canada.

Sung, Gi-Ho, George O. Poinar, and Joseph W. Spatafora. "The oldest fossil evidence of animal parasitism by fungi supports a Cretaceous diversification of fungal-arthropod symbioses." Molecular phylogenetics and evolution 49.2 (2008): 495-502.

Thomas, F., Poulin, R., de Meeüs, T., Guégan, J.F. and Renaud, F., 1999. Parasites and ecosystem engineering: what roles could they play?. Oikos , pp.167-171.

Thomas, F., Adamo, S. and Moore, J., 2005. Parasitic manipulation: where are we and where should we go?. Behavioural processes , 68 (3), pp.185-199.

Weinersmith, Kelly, and Zen Faulkes. "Parasitic manipulation of hosts' phenotype, or how to make a zombiean introduction to the symposium." Integrative and comparative biology 54.2 (2014): 93-100.

Windsor, Donald A. "Controversies in parasitology, Most of the species on Earth are parasites." International journal for parasitology28.12 (1998): 1939-1941. 


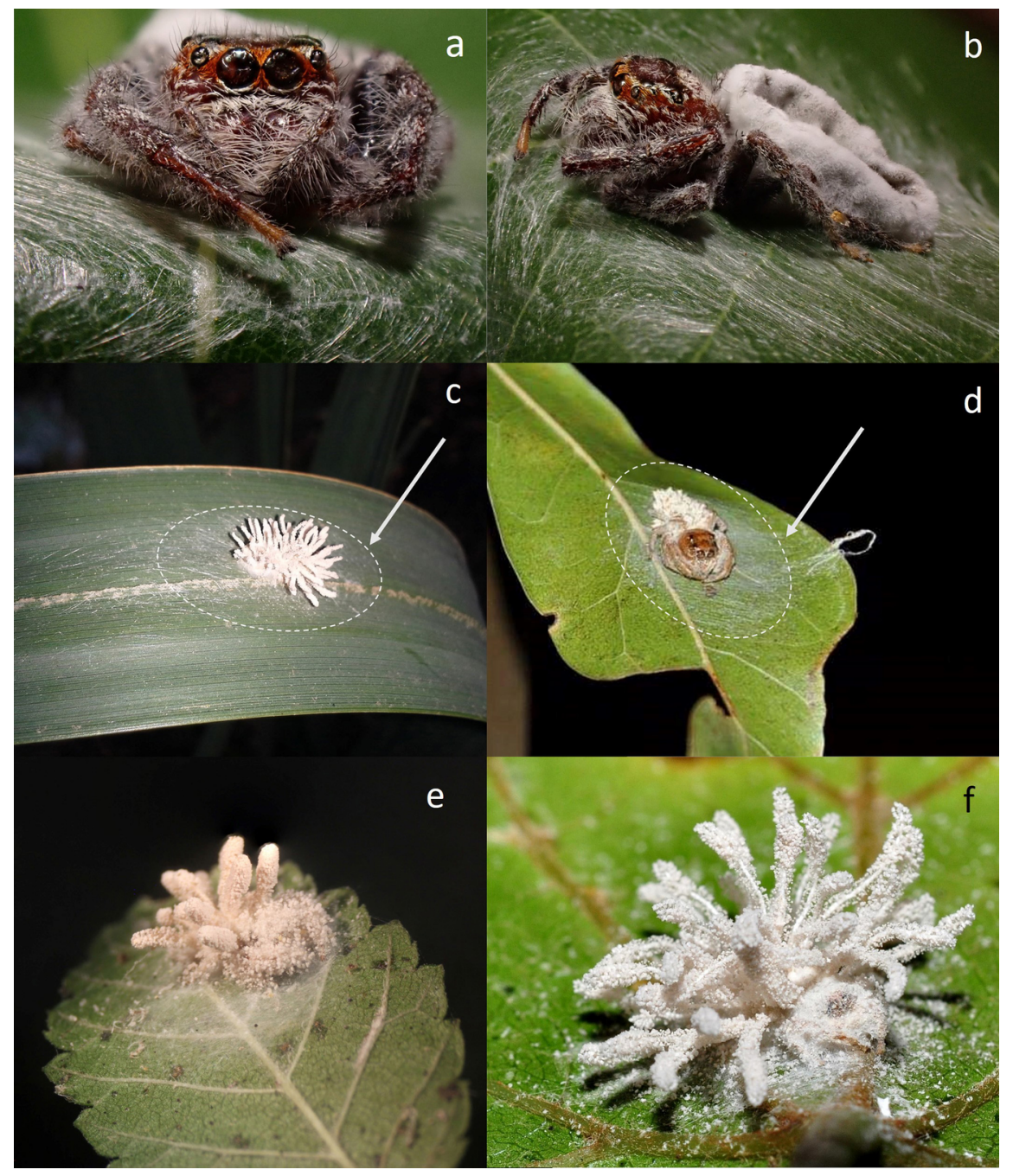



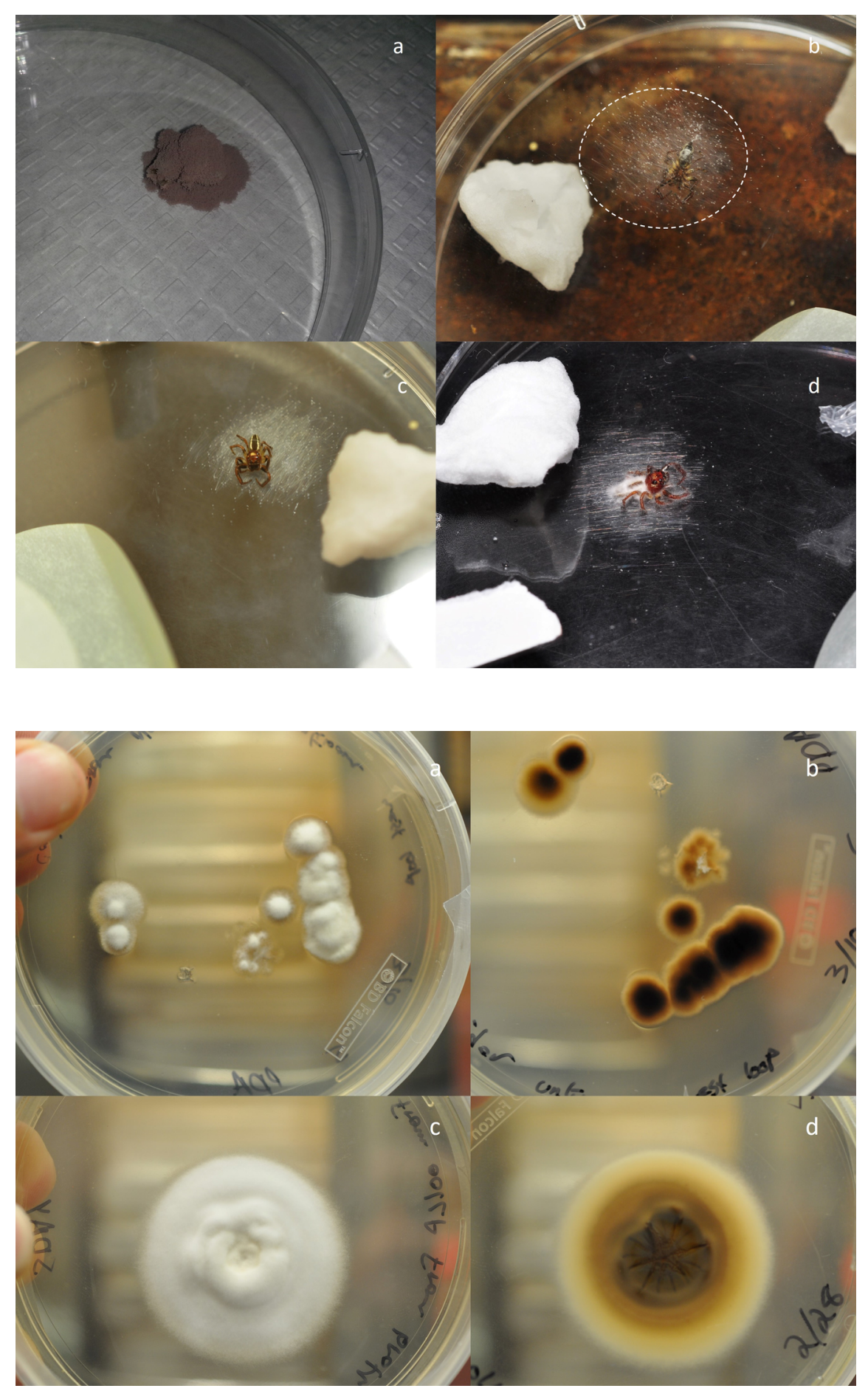